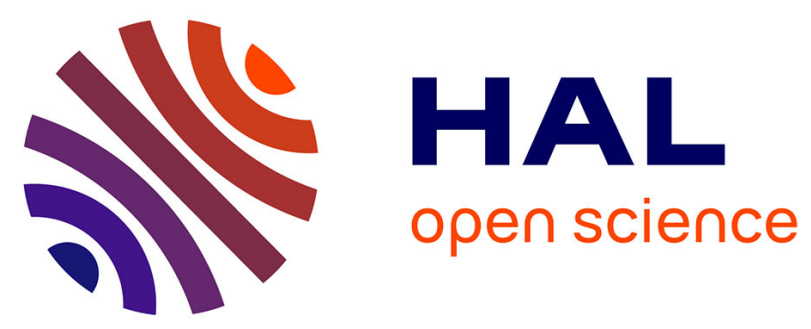

\title{
An iterative method for the Cauchy problem in linear elasticity with fading regularization effect
}

\author{
Franck Delvare, Alain Cimetière, Jean-Luc Hanus, Patrice Bailly
}

\section{To cite this version:}

Franck Delvare, Alain Cimetière, Jean-Luc Hanus, Patrice Bailly. An iterative method for the Cauchy problem in linear elasticity with fading regularization effect. Computer Methods in Applied Mechanics and Engineering, 2010, 199 (49-52), pp.3336-3344. 10.1016/j.cma.2010.07.004 . hal-00601541

\section{HAL Id: hal-00601541 \\ https://hal.science/hal-00601541}

Submitted on 20 Jun 2011

HAL is a multi-disciplinary open access archive for the deposit and dissemination of scientific research documents, whether they are published or not. The documents may come from teaching and research institutions in France or abroad, or from public or private research centers.
L'archive ouverte pluridisciplinaire HAL, est destinée au dépôt et à la diffusion de documents scientifiques de niveau recherche, publiés ou non, émanant des établissements d'enseignement et de recherche français ou étrangers, des laboratoires publics ou privés. 


\title{
An iterative method for the Cauchy problem in linear elasticity with fading regularization effect
}

\author{
Franck DELVARE ${ }^{\mathrm{a}}$, Alain CIMETIÈRE ${ }^{\mathrm{b}}$, Jean-Luc HANUS ${ }^{\mathrm{a}}$, Patrice BAILLY ${ }^{\mathrm{a}}$ \\ ${ }^{a}$ Institut PRISME, ENSI de Bourges, 88, Boulevard Lahitolle, 18020 Bourges Cedex, France \\ ${ }^{b}$ Institut Pprime, CNRS-ENSMA-Université de Poitiers, UPR 3346, Boulevard Marie et Pierre Curie, BP 30179, 86962 Chasseneuil Futuroscope Cedex, France
}

\begin{abstract}
In this paper, an iterative method for solving the Cauchy problem in linear elasticity is introduced. This problem consists in recovering missing data (displacements and forces) on some parts of a domain boundary from the knowledge of overspecified data (displacements and forces) on the remaining parts. The algorithm reads as a least square fitting of the given data, with a regularization term whose effect fades as the iterations go on. So the algorithm converges to the solution of the Cauchy problem. Numerical simulations using the finite element method highlight the algorithm's efficiency, accuracy, robustness to noisy data as well as its ability to deblur noisy data.
\end{abstract}

Key words:

Cauchy problem, Inverse problem, Data completion, Linear elasticity, Regularization, Boundary conditions

\section{Introduction}

Inverse problems arise in many engineering fields such as for instance in thermal sciences, electrostatics, solid and fluid mechanics. Kubo [24] has defined inverse problems in opposition to direct problems. Direct problems are problems for which the geometry of the domain, the equilibrium equations, the constitutive equations, the boundary conditions on the whole boundary of the domain and the initial conditions are all given. For Kubo, inverse problems can be characterized by the lack of at least one of these pieces of information. According to this definition, many mechanical problems, for example, identification of material parameters, identification of unknown boundaries (such as contact zones, cavities or cracks), identification of residual stresses, identification of initial boundary conditions, identification of inaccessible boundary conditions can be considered as inverse problems. Some examples arising from fracture mechanics can be found in [6] and some relating to elasticity problems in [4]. In a mathematical sense, direct problems can be considered as well-posed problems. In linear cases, these problems have a unique solution which is stable (continuously dependent on the data). Conversely, inverse problems are generally ill-posed problems in the Hadamard sense [17], since existence or uniqueness or continuous dependence on the data of their solutions may not be ensured.

This paper examines an inverse boundary value problem in linear elasticity. It consists in recovering missing data (both displacement and traction vectors are unknown) on some part of the boundary of a domain from overspecified data (both displacement and traction vectors are given) on the other part. In this case, the equilibrium equations, the constitutive equations, the domain and its boundary are known. This problem is namely known as a Cauchy problem and is a data completion problem. In order to solve Cauchy problems for elliptic equations, many regularization methods have been introduced. The references $[1,5,8,9,11,12,14,20,21,23,25,26]$ propose different methods of solving the Cauchy problem for the Laplace equation. References $[2,3,13,22,23,27,28,29,30,31,32$, $33,34,35,36,37,38,40,41]$ deal with the Cauchy problem in linear elasticity. These methods can be classified as Tikhonov type methods [15, 22, 27, 29, 33, 34, 35, 38, 39, 40, 41], quasi-reversibility type methods $[5,21,25]$, iterative methods $[1,2,3,8,9,11,12,13,14,15,20,23,26,28,32,36,37], \ldots$ Quasi reversibility methods and Tikhonov regularization methods present the advantage of leading to well posed problems after modifying the equilibrium equations. Some iterative methods are based on the use of a sequence of well-posed problems and others on the minimization of an energy-like functional. Numerical algorithms are implemented using different numerical methods, such as the finite element method (FEM) [1, 2, 3, $5,8,9,11,13,27,38]$, the boundary element method (BEM) $[12,14,20,22,26,23,28,29,30,31,32,33,36,37,40,41]$, the finite difference method [21] or meshless methods [34, 35]. Some papers present comparisons between different numerical methods [10, 31, 37].

In previous works $[8,9,12]$ a somewhat different resolution approach was first introduced in [8] for the Cauchy problem associated with the Laplace equation. This approach reduced the resolution of the Cauchy problem to the resolution of a sequence of optimization problems under equality constraints. The functional is composed of two terms. At each step of the resolution, the first term gives the gap between the optimal element and the overspecified boundary data (relaxation term), the second one the gap between the optimal element and the 
previous optimal element (regularization term). The equality constraints are the equilibrium equations. So, at each step an optimal element is obtained which is an exact solution to the equilibrium equations and is nearer to the overspecified data than the previous optimal element calculated. In the case of compatible data, it was also proved that the sequence converges and its limit is the solution to the Cauchy problem. The additional regularization term tends to zero as iterations continue. It is the reason why this method is called the evanescent regularization method.

In this paper, this inverse technique is extended to the Cauchy problem in linear elasticity. The next section is devoted to the formulation of the Cauchy problem in linear elasticity. The third section describes the iterative inverse method and the fourth section is devoted to its numerical implementation using the finite element method. In the fifth section, several numerical simulations are presented.

\section{The Cauchy problem in linear elasticity}

Let us consider a linear elastic material which occupies an open set $\Omega$ in $\mathbb{R}^{2}$ or $\mathbb{R}^{3}$, with a smooth boundary $\Gamma$, assuming that the boundary is divided in two parts $\Gamma_{d}$ and $\Gamma_{u}$, where $\Gamma_{d} \cup$ $\Gamma_{u}=\Gamma$ and $\Gamma_{d} \cap \Gamma_{u}=\varnothing$. The subscript $d$ denotes the data and the subscript $u$ denotes the unknowns. With no body force, the equilibrium equations are given by:

$$
\frac{\partial \sigma_{i j}(x)}{\partial x_{j}}=0 \quad x \in \Omega
$$

where $\sigma_{i j}$ is the Cauchy stress tensor. This tensor is energy conjugated to the infinitesimal strain tensor $\varepsilon_{i j}$ by the following constitutive equations:

$$
\sigma_{i j}=2 \mu \varepsilon_{i j}+\lambda \delta_{i j} \varepsilon_{k k}
$$

where $\lambda$ and $\mu$ are the Lamé constants. These are related to Young's modulus $E$ and Poisson's ratio $v$ as:

$$
\begin{gathered}
\lambda=\frac{v E}{(1+v)(1-2 v)} \\
\mu=\frac{E}{2(1+v)}
\end{gathered}
$$

The linear strain tensor conponents $\varepsilon_{i j}$, as function of the displacement gradients, are given by:

$$
\varepsilon_{i j}=\frac{1}{2}\left(\frac{\partial u_{j}}{\partial x_{i}}+\frac{\partial u_{i}}{\partial x_{j}}\right)
$$

By substituting Hooke's law (2) into the governing equations (1), the Lamé equations are obtained:

$$
[\mathbf{L}(u)]_{i}=\frac{E}{2(1+v)} \frac{\partial^{2} u_{i}}{\partial x_{j}^{2}}+\frac{E}{2(1+v)(1-2 v)} \frac{\partial^{2} u_{j}}{\partial x_{i} \partial x_{j}}=0
$$

At a point $x \in \Gamma, n(x)$ the outward unit normal vector is defined as well as the traction vector $p(x)$ whose components are defined by:

$$
p_{i}(x)=p_{i}(u(x))=\sigma_{i j}(u(x)) n_{j}(x) \quad x \in \Gamma
$$

It is assumed that both the displacement vector and the traction vector are given or known on the part of boundary $\Gamma_{d}$ but no condition is prescribed on the remaining part $\Gamma_{u}$ :

$$
\begin{array}{ll}
u(x)=\phi^{d} & x \in \Gamma_{d} \\
p(x)=\psi^{d} & x \in \Gamma_{d}
\end{array}
$$

where $\phi^{d}$ and $\psi^{d}$ are prescribed vector functions. The Lamé (or Navier) system (4) and the boundary conditions (5) lead to the formulation of the Cauchy problem in linear elasticity:

$$
\left\{\begin{array}{lc}
\mathbf{L}(u)=0 & x \in \Omega \\
u(x)=\phi^{d} & x \in \Gamma_{d} \\
p(x)=\psi^{d} & x \in \Gamma_{d}
\end{array}\right.
$$

This problem is difficult to solve, since it is ill-posed. When it admits a solution, its solution is unique [40], but it is known to be very sensitive [17] to small perturbations on boundary conditions (5).

\section{The evanescent regularization method}

Let us introduce the space $H(\Omega)$ of solutions of the equilibrium equation (4):

$$
H(\Omega)=\{v \text { satisfying } \mathbf{L}(v)=0 \text { in } \Omega\}
$$

Next, let us denote $H(\Gamma)$ the space composed of couples of restrictions on $\Gamma$ of elements $v$ in $H(\Omega)$ and of their associated tractions $p(v)$. An equivalent formulation of problem (6) reads:

$$
\left\{\begin{array}{l}
\text { Find } \mathbf{U}=(u, p) \in H(\Gamma) \text { such as : } \\
\mathbf{U}=\boldsymbol{\Phi}^{\mathbf{d}} \text { on } \Gamma_{d}
\end{array}\right.
$$

where $\boldsymbol{\Phi}^{\mathbf{d}}=\left(\phi^{d}, \psi^{d}\right)$.

The problem (7) is also ill-posed even if it admits a unique solution. So then an iterative regularizing method is introduced to solve it. This method is a generalization of the inverse technique introduced by Cimetière et al $[8,9]$ to solve the Cauchy problem for the Laplace equation. It can be considered as an iterative Tikhonov-type method. Given $c>0$ and $\mathbf{U}^{\mathbf{0}} \in H(\Gamma)$ the iterative algorithm reads:

$$
\mid \begin{aligned}
& \text { Find } \mathbf{U}^{\mathbf{k}+\mathbf{1}} \in H(\Gamma) \text { such as : } \\
& J_{c}^{k}\left(\mathbf{U}^{\mathbf{k}+\mathbf{1}}\right) \leq J_{c}^{k}(\mathbf{V}) \quad \forall \mathbf{V} \in H(\Gamma) \text { with } \\
& J_{c}^{k}(\mathbf{V})=\left\|\mathbf{V}-\boldsymbol{\Phi}^{\mathbf{d}}\right\|_{\Gamma_{d}}^{2}+c\left\|\mathbf{V}-\mathbf{U}^{\mathbf{k}}\right\|_{\Gamma}^{2}
\end{aligned}
$$

where the norms are defined by:

$$
\begin{aligned}
\|\mathbf{V}\|_{\Gamma_{d}}^{2} & =\int_{\Gamma_{d}} v^{2} d s+\frac{1}{E^{2}} \int_{\Gamma_{d}} p^{2} d s \\
\|\mathbf{V}\|_{\Gamma}^{2} & =\int_{\Gamma} v^{2} d s+\frac{1}{E^{2}} \int_{\Gamma} p^{2} d s
\end{aligned}
$$

In this iterative process, the equilibrium equation (4) is taken into account exactly since at each step the search for the optimal element is performed in space $H(\Gamma)$. The functional is composed of two terms which play different roles. The 
first one acts only on $\Gamma_{d}$ and represents the gap between the optimal element and the overspecified boundary data. It relaxes the overspecified data which can be possibly blurred by measurement noises (relaxation term). The second one acts on the whole boundary $\Gamma$ and not only on the boundary $\Gamma_{u}$ where the boundary conditions are to be completed. This term is a regularization term and controls the distance between the new optimal element and the previous optimal element. This term tends to zero as iterations go on.

So, at each step the optimal element obtained is an exact solution of the equilibrium equation (4) and is near to the overspecified data $\boldsymbol{\Phi}^{\mathbf{d}}=\left(\phi^{d}, \psi^{d}\right)$. This unique optimal element $\mathbf{U}^{\mathbf{k}+\mathbf{1}}$ is characterized by:

$$
\left\langle\mathbf{U}^{\mathbf{k}+\mathbf{1}}-\boldsymbol{\Phi}^{\mathbf{d}}, \mathbf{V}\right\rangle_{\Gamma_{d}}+c\left\langle\mathbf{U}^{\mathbf{k}+\mathbf{1}}-\mathbf{U}^{\mathbf{k}}, \mathbf{V}\right\rangle_{\Gamma}=0 \forall \mathbf{V} \in H(\Gamma)
$$

\section{Theorem: Convergence of the sequence}

Let $\boldsymbol{\Phi}^{\mathbf{d}}$ be compatible data associated with the compatible pair $\mathbf{U}_{\mathbf{e}} \in H(\Gamma)$. Then the sequence produced by the iterative scheme (8) strongly converges to $\boldsymbol{\Phi}^{\mathbf{d}}$ on $\Gamma_{d}$ and weakly to $\mathbf{U}_{\mathbf{e}}$ on $\Gamma$.

The proof of this theorem is based on the following lemma.

\section{LEMMA:}

$\overline{\text { For all }} n \in \mathbb{N}$, the sequence $\left(\mathbf{U}^{\mathbf{k}}\right)$ corresponding of optimal elements verifies:

$$
\begin{aligned}
& \left\|\mathbf{U}^{\mathbf{n}+\mathbf{1}}-\mathbf{U}_{\mathbf{e}}\right\|_{\Gamma}^{2}+\sum_{k=0}^{n}\left\|\mathbf{U}^{\mathbf{k}+\mathbf{1}}-\mathbf{U}^{\mathbf{k}}\right\|_{\Gamma}^{2}+ \\
& \frac{2}{c} \sum_{k=0}^{n}\left\|\mathbf{U}^{\mathbf{k}+\mathbf{1}}-\boldsymbol{\Phi}^{\mathbf{d}}\right\|_{\Gamma_{d}}^{2}=\left\|\mathbf{U}^{\mathbf{0}}-\mathbf{U}_{\mathbf{e}}\right\|_{\Gamma}^{2}
\end{aligned}
$$

where $\mathbf{U}_{\mathbf{e}}$ is the solution of Cauchy problem (6) and $\boldsymbol{\Phi}^{\mathbf{d}}=\left.\mathbf{U}_{\mathbf{e}}\right|_{\Gamma_{d}}$. The proof of the lemma and consequently those of the theorem is similar to that established for the algorithm introduced in [8] to solve the Cauchy problem associated with the Laplace equation. This proof is valid for all $c>0$. The $c$ value only influences the convergence rate of the algorithm.

\section{The implementation using the Finite Element Method}

The implementation of the inverse method using the finite element method is presented in 2D situations.

\subsection{Discrete solutions space}

The first issue in this section is to discretize space $H(\Gamma)$. Our main concern has been to make use of any ordinary finite element code, not to work out a specific one. As a matter of fact, computations were run using $\operatorname{Cast}_{3 \mathrm{M}^{1}}$ [7] and piecewise linear finite elements, which means a piecewise constant approximation for the traction vector. Let us now discretize the domain $\Omega$,

${ }^{1}$ The FE code Cast3M is developed by the Department of Mechanics and Technology (DMT) of the French Atomic Energy Agency (CEA DEN/DM2S/SEMT), http://www-cast3m.cea.fr $h$ being the discretization parameter standing for the element size, leading to $n$ nodes and $n$ elements on the boundary, and $m$ nodes inside the domain. Let $V_{h}$ be the space of continuous piecewise linear functions with respect to the mesh, and let us define $V_{h}(\Gamma)$ and $W_{h}(\Gamma)$ as the space of continuous piecewise linear functions and the space of piecewise constant functions on the boundary. Traces of functions belonging to $V_{h}(\Omega)$ span the space $V_{h}(\Gamma)$, whereas the associated traction vectors belong to the space $W_{h}(\Gamma)$ of piecewise constant functions. Defining $U$ and $P$ as the $2 n$ vectors standing respectively for the $2 n$ nodal values of $u$ and the $2 n$ discrete values of $p$ on the boundary, and $U^{*}$ the $2 m$-vector of internal nodal values of $u$, the discrete equilibrium equations read as:

$$
\left[\begin{array}{ll}
A_{i i} & A_{e i}^{T} \\
A_{e i} & A_{e e}
\end{array}\right]\left(\begin{array}{l}
U^{*} \\
U
\end{array}\right)=\left(\begin{array}{l}
0 \\
-B P
\end{array}\right)
$$

$A_{i i}$ is the stiffness matrix corresponding to the Dirichlet problem and thus is invertible. Expressing the internal unknowns $U^{*}$ in terms of the boundary ones $U$, i.e performing a condensation, equation (11) reduces to:

$$
\left(A_{e e}-A_{e i} A_{i i}^{-1} A_{e i}^{T}\right) U+B P=0
$$

The matrix form of (12) reads:

$$
\left[\begin{array}{ll}
A & B
\end{array}\right]\left(\begin{array}{l}
U \\
P
\end{array}\right)=0
$$

The finite element method leads to the definition of the following discrete compatible pairs space which reads:

$$
H_{h}(\Gamma)=\left\{\begin{array}{l}
(U, P) \in \mathbb{R}^{2 n} \times \mathbb{R}^{2 n} \text { such that } \\
E_{h}(U, P)=A U+B P=0
\end{array}\right\}
$$

where $E_{h}$ denotes a linear operator mapping $\mathbb{R}^{2 n} \times \mathbb{R}^{2 n}$ onto $\mathbb{R}^{2 n}$.

\subsection{Discretization of the $(k+1)^{\text {th }}$ iteration}

Given now $c>0$ and $\left(U^{k}, P^{k}\right) \in H_{h}(\Gamma)$, iteration $(k+1)$ of the discretized iterative algorithm reads as follows:

Find $\left(U^{k+1}, P^{k+1}\right) \in \mathbb{R}^{2 n} \times \mathbb{R}^{2 n}$ such that : $J_{c}^{k}\left(U^{k+1}, P^{k+1}\right) \leq J_{c}^{k}(V, Q) \quad \forall(V, Q) \in \mathbb{R}^{2 n} \times \mathbb{R}^{2 n}$ under the $2 n$ scalar equality constraints $E_{h}(V, Q)=0$

Problem (15) is a minimization problem in $R^{2 n} \times R^{2 n}$ under the $2 n$ equality constraints expressed by (13). Its solution is given by:

$$
\mid \begin{aligned}
& \text { Initializing with }\left(U^{0}=0, P^{0}=0\right) \\
& \text { Find }\left(U^{k+1}, P^{k+1}, \lambda^{k+1}\right) \in \mathbb{R}^{2 n} \times \mathbb{R}^{2 n} \times \mathbb{R}^{2 n} \text { such that : } \\
& \nabla J_{c}^{k}\left(U^{k+1}, P^{k+1}\right)+\left(\lambda^{k+1}\right)^{T} \nabla E_{h}\left(U^{k+1}, P^{k+1}\right)=0 \\
& E_{h}\left(U^{k+1}, P^{k+1}\right)=0
\end{aligned}
$$

where $\lambda^{k+1}$ is a $2 n$-vector of Lagrange multipliers introduced to take the equality constraints (13) into account. Each iteration 
in the iterative algorithm needs to solve a system of $6 n$ linear equations with $6 n$ unknowns. The matrix of this linear system is independant of the iterations and needs to be computed only once. For this reason, a direct algorithm (the Crout factorization) has been preferred to iterative methods. The factorization, which is obtained at the first step, is also used at each following step.

In the case of compatible data, the proof of the convergence of the discrete algorithm is similar to that established for the corresponding algorithm used to solve the Cauchy problem for the Laplace equation [8]. This proof is valid for all $c>0$ and the $c$ value only influences the convergence rate of the algorithm.

\section{Numerical results}

The purpose of this section is to present numerical results obtained with the method introduced. Firstly, different cases are considered for which an analytical solution $u^{a n}$ is known. These cases have been previously studied, among others, by [28, 30, 37].

The following control quantities are used to estimate the accuracy of the method and to determine a stopping criteria for the iterative process:

- the norm of the error made on $u$ :

$$
u_{\text {error }}=\frac{\int_{\Gamma}\left(U-u^{a n}\right)^{2} d s}{\int_{\Gamma}\left(u^{a n}\right)^{2} d s}
$$

- the norm of the error made on $p$ :

$$
p_{\text {error }}=\frac{\int_{\Gamma}\left(P-p^{a n}\right)^{2} d s}{\int_{\Gamma}\left(p^{a n}\right)^{2} d s}
$$

- the first term (relaxation term) of the functional $J_{\Gamma_{d}}\left(\mathbf{U}^{\mathbf{k}+\mathbf{1}}\right)=\left\|\mathbf{U}^{\mathbf{k}+\mathbf{1}}-\boldsymbol{\Phi}^{\mathbf{d}}\right\|_{\Gamma_{d}}^{2}$

- the second term (regularization term) of the functional $J_{\Gamma}\left(\mathbf{U}^{\mathbf{k}+\mathbf{1}}\right)=c\left\|\mathbf{U}^{\mathbf{k}+\mathbf{1}}-\mathbf{U}^{\mathbf{k}}\right\|_{\Gamma}^{2}$

- and the value $J\left(\mathbf{U}^{\mathbf{k}+\mathbf{1}}\right)=J_{\Gamma_{d}}\left(\mathbf{U}^{\mathbf{k}+\mathbf{1}}\right)+J_{\Gamma}\left(\mathbf{U}^{\mathbf{k}+\mathbf{1}}\right)$ of the functional for the optimal element $\mathbf{U}^{\mathbf{k}+\mathbf{1}}$.

Three properties of the functional terms in the minimizing sequence can be easily established without the assumption that the data $\boldsymbol{\Phi}^{\mathbf{d}}$ is compatible:

- The first term of the functional $J_{\Gamma_{d}}\left(\mathbf{U}^{\mathbf{k}}\right)$ is monotonically decreasing :

$$
\left\|\mathbf{U}^{\mathbf{k}+\mathbf{1}}-\boldsymbol{\Phi}^{\mathbf{d}}\right\|_{\Gamma_{d}}^{2} \leq\left\|\mathbf{U}^{\mathbf{k}}-\boldsymbol{\Phi}^{\mathbf{d}}\right\|_{\Gamma_{d}}^{2}
$$

- The second one $J_{\Gamma}\left(\mathbf{U}^{\mathbf{k}}\right)$ is monotonically decreasing as soon as $c>0$ :

$$
\left\|\mathbf{U}^{\mathbf{k}+\mathbf{1}}-\mathbf{U}^{\mathbf{k}}\right\|_{\Gamma}^{2} \leq\left\|\mathbf{U}^{\mathbf{k}}-\mathbf{U}^{\mathbf{k}-\mathbf{1}}\right\|_{\Gamma}^{2}
$$

- The sequence defined by the values of the functional $J$ for each optimal element $\mathbf{U}^{\mathbf{k}}$ is also monotonically decreasing as soon as $c>0$ :

$$
J\left(\mathbf{U}^{\mathbf{k}+\mathbf{1}}\right) \leq J\left(\mathbf{U}^{\mathbf{k}}\right)
$$

In the last example, the compression of an infinite cylinder between two rigid planes is investigated and shows that the iterative inverse method allows us to reconstruct the contact zones and the contact pressure distribution from the knowledge of the displacement on a part of the free force boundary.

The procedure used during the numerical simulations is as follows:

- The meshing of the boundary is made using SEG2 elements. The SEG2 element is a finite element with two nodes which leads to a linear interpolation of the displacements. This induces a piecewise constant interpolation of the traction vector components.

- The user specifies the meshing of the boundary specifying the number and the distribution of the finite elements on each part of the boundary.

- Then, the mesh of the entire domain is generated automatically by a routine included in the software Cast3m [7]. This mesh is constituted of 4-node quadrilaterals and 3node triangles.

- The computation and the assembly of the stiffness matrix corresponding to the domain is performed thanks to the standard routines of Cast $3 \mathrm{~m}$ software [7].

- This software creates a superelement based on the boundary and computes the corresponding stiffness matrix. This leads to the condensed stiffness matrix A.

- The stiffness matrix $\mathrm{A}$ is then used by the specific code that implements the inverse method introduced.

Note that the inverse method only involves values on the boundary. Therefore, for the different numerical tests only the discretization of the boundary $\Gamma$ will be specified. Moreover, note that all numerical computations have been performed on a machine with a $2.20 \mathrm{GHz}$ Intel ${ }^{\circledR}$ Core $^{T M} 2$ Duo processor $\mathrm{T} 7500$.

\subsection{Hollow cylinder under internal and external uniform pres- sures: Reconstruction on the inner boundary}

A two-dimensional isotropic linear elastic medium in a plane stress state characterized by the material constants $E=97.9$ $G P a$ and $v=0.34$ corresponding to a copper alloy is studied. The annular domain $\Omega$ is defined by:

$$
\begin{aligned}
& \Omega=\left\{x=\left(x_{1}, x_{2}\right) / r_{i}^{2}<x_{1}^{2}+x_{2}^{2}<r_{0}^{2}\right\} \\
& \text { with } r_{i}=1 \mathrm{~m} \text { and } r_{0}=4 \mathrm{~m} .
\end{aligned}
$$

The boundary part $\Gamma_{d}$ is defined by:

$$
\Gamma_{d}=\left\{x=\left(x_{1}, x_{2}\right) \in \Gamma / x_{1}^{2}+x_{2}^{2}=r_{0}^{2}\right\}
$$


$\Gamma_{d}$ is discretized using $N$ finite elements SEG2. All finite elements have the same length and the nodes are uniformly distributed. The boundary part $\Gamma_{u}$ is defined by:

$$
\Gamma_{u}=\left\{x=\left(x_{1}, x_{2}\right) \in \Gamma / x_{1}^{2}+x_{2}^{2}=r_{i}^{2}\right\}
$$

and discretized using a regular mesh with $N$ finite elements SEG2. It is used to find the displacement and the pressure on $\Gamma_{u}$, from the knowledge of the displacement and the pressure on $\Gamma_{d}$. The data are built using the analytical solution for the displacements:

$$
u_{j}^{a n}\left(x_{1}, x_{2}\right)=\left(V \frac{1-v}{E} x_{j}-W \frac{1+v}{E} \frac{x_{j}}{x_{1}^{2}+x_{2}^{2}}\right) x_{j}
$$

where

$$
j=1,2 ; \quad V=-\frac{\sigma_{0} r_{0}^{2}-\sigma_{i} r_{i}^{2}}{r_{0}^{2}-r_{i}^{2}} ; \quad W=\frac{\left(\sigma_{0}-\sigma_{i}\right) r_{0}^{2} r_{i}^{2}}{r_{0}^{2}-r_{i}^{2}}
$$

with $\sigma_{i}=10 \mathrm{GPa}$ and $\sigma_{0}=20 \mathrm{GPa}$, which corresponds to constant internal and external pressures for which the stress tensor is given by:

$$
\begin{aligned}
& \sigma_{11}\left(x_{1}, x_{2}\right)=V+W \frac{x_{1}^{2}-x_{2}^{2}}{\left(x_{1}^{2}+x_{2}^{2}\right)^{2}} \\
& \sigma_{22}\left(x_{1}, x_{2}\right)=V-W \frac{x_{1}^{2}-x_{2}^{2}}{\left(x_{1}^{2}+x_{2}^{2}\right)^{2}} \\
& \sigma_{12}\left(x_{1}, x_{2}\right)=2 W \frac{x_{1} x_{2}}{\left(x_{1}^{2}+x_{2}^{2}\right)^{2}} .
\end{aligned}
$$

\subsubsection{Stopping criterion}

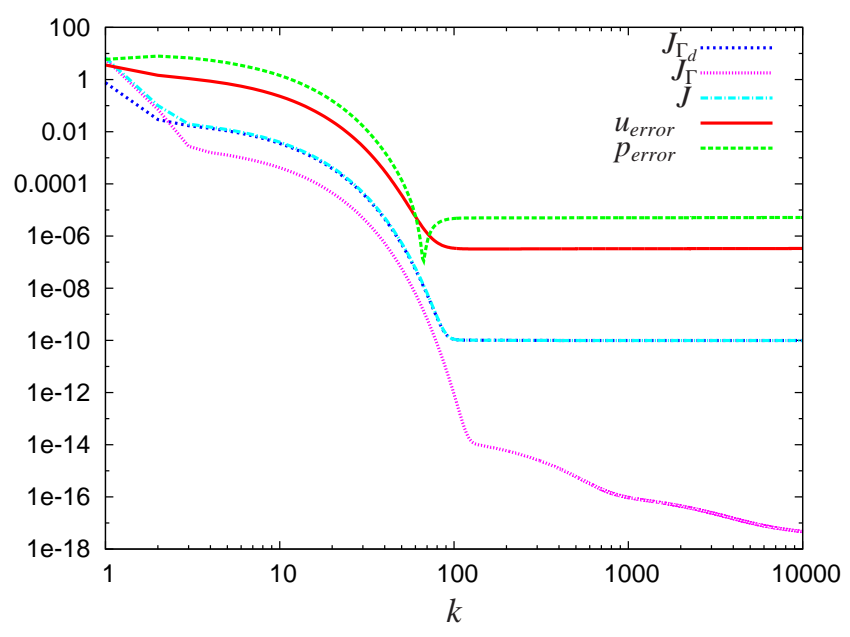

Figure 1: evolution of control quantities versus the number of iterations $k$

In a first step, a reliable stopping criterion is needed to stop the iterative process. In the present case, the data on $\Gamma_{d}$ are generated from the analytical solution (equations (20) and (22)) and are not blurred by measurement noise. The boundaries $\Gamma_{d}$ and $\Gamma_{u}$ are both approximated with 160 SEG2 finite elements.
The value of the parameter $c$ is arbitrarily fixed at 0.1 . Figure 1 represents the evolution of different control quantities $J\left(\mathbf{U}^{\mathbf{k}+\mathbf{1}}\right)$, $J_{\Gamma_{d}}\left(\mathbf{U}^{\mathbf{k}+\mathbf{1}}\right), J_{\Gamma}\left(\mathbf{U}^{\mathbf{k}+\mathbf{1}}\right), u_{\text {error }}$ and $p_{\text {error }}$ versus the number of iterations $k$. We notice from the $130^{\text {th }}$ iteration that the quantities $u_{\text {error }}, p_{\text {error }}, J\left(\mathbf{U}^{\mathbf{k}+\mathbf{1}}\right)$ and $J_{\Gamma_{d}}\left(\mathbf{U}^{\mathbf{k}+\mathbf{1}}\right)$ remain constant. This confirms that the method converges. The determination of the iteration to stop the iterative process is made using the quantity $J\left(\mathbf{U}^{\mathbf{k}+\mathbf{1}}\right)$ where $\mathbf{U}^{\mathbf{k}+\mathbf{1}}$ is the optimal element obtained at the $(k+1)^{t h}$ step. The evolution of $J$ follows an L-curve [18]. As expected from relation (19), the control quantity $J$ decreases when $k<130$ and then becomes almost constant when $k>130$ and never reincreases. So the iterative process is stopped when $k=130$. This stopping criterion is blind because when calculating the quantity $J$ it does not need to know the analytical solution. Indeed, it only needs to know two successive optimal elements and the data on $\Gamma_{d}$. As expected from relation (18), we may also notice that $J_{\Gamma}$ (regularization term) decreases as iterations go on. This term becomes negligible compared to $J_{\Gamma_{d}}$ and tends to zero. This proves that the algorithm converges. As expected from relation (17), the residual error $J_{\Gamma_{d}}$ (relaxation term) decreases during the iterative process. After convergence this term remains constant and corresponds to the approximation error of the finite element method.

It can also be observed that the errors in the numerical tractions obtained $\left(p_{\text {error }}\right)$ using the iterative method are larger than those corresponding to the reconstructed displacements $\left(u_{\text {error }}\right)$. This last remark is also valid for all the following numerical examples which will be analysed.

\subsubsection{Influence of parameter $c$}

It is necessary to look at the influence of parameter $c$ which defines the relative weight of the regularization term $J_{\Gamma}$ compared to the relaxation term $J_{\Gamma_{d}}$. For the same discretization as in the previous example, different values of the parameter $c$ are tested. Table 1 lists the results obtained for each value of parameter $c$ by specifying the number of iterations necessary to achieve convergence, the $u_{\text {error }}$, the $p_{\text {error }}$ and the CPU time. The errors on $u$ and $p$ are quite identical for each value of the parameter $c$. This confirms that the algorithm converges to the

\begin{tabular}{|l|l|l|l|l|}
\hline$c$ & $k$ & $u_{\text {error }}$ in $\%$ & $p_{\text {error }}$ in $\%$ & CPU time in $s$ \\
\hline 10. & 12810 & $3.20 \mathrm{E}-7$ & $4.95 \mathrm{E}-6$ & 59.93 \\
\hline 1. & 1278 & $3.20 \mathrm{E}-7$ & $4.95 \mathrm{E}-6$ & 8.61 \\
\hline 0.1 & 130 & $3.20 \mathrm{E}-7$ & $4.95 \mathrm{E}-6$ & 3.50 \\
\hline 0.01 & 20 & $3.21 \mathrm{E}-7$ & $4.96 \mathrm{E}-6$ & 3.01 \\
\hline 0.001 & 5 & $3.28 \mathrm{E}-7$ & $4.95 \mathrm{E}-6$ & 2.95 \\
\hline 0.0001 & 3 & $3.29 \mathrm{E}-7$ & $5.05 \mathrm{E}-6$ & 2.94 \\
\hline 0.00001 & 2 & $3.36 \mathrm{E}-7$ & $5.22 \mathrm{E}-6$ & 2.94 \\
\hline
\end{tabular}

Table 1: influence of $c$ on the number of iterations $k$ to achieve convergence influence of $c$ on the $u_{\text {error }}$ and on the perror

same solution whatever the value of $c$. However the choice of the parameter $c$ affects the number $k$ of iterations needed to obtain convergence. The evolution of the number of iterations necessary to achieve convergence seems to evolve linearly with 
$c$ where $0.1<c<10$. From the evolution of the CPU time with $c$ (or with the number of iterations $k$ ) it can be deduced that the CPU time taken by each iteration for $k>1$ is roughly $4.410^{-3}$ $s$. This CPU time is lower than the CPU time taken to achieve both the preliminary computations and the first iteration (roughly $2.93 s$ ). For a small value of $c$, the convergence only takes a few iterations but the $p_{\text {error }}$ increases a little. This may be explained by the fact that the regularization term becomes too weak and induces some instabilities on the reconstructions. Moreover, it is preferable to have a greater value of the parameter $c$ which induces few additional CPU time in order to have more accurate reconstructions. Subsequently, the value of $c$ and the number of iterations required to achieve convergence will be no longer specified.

\subsubsection{Influence of mesh refinement}

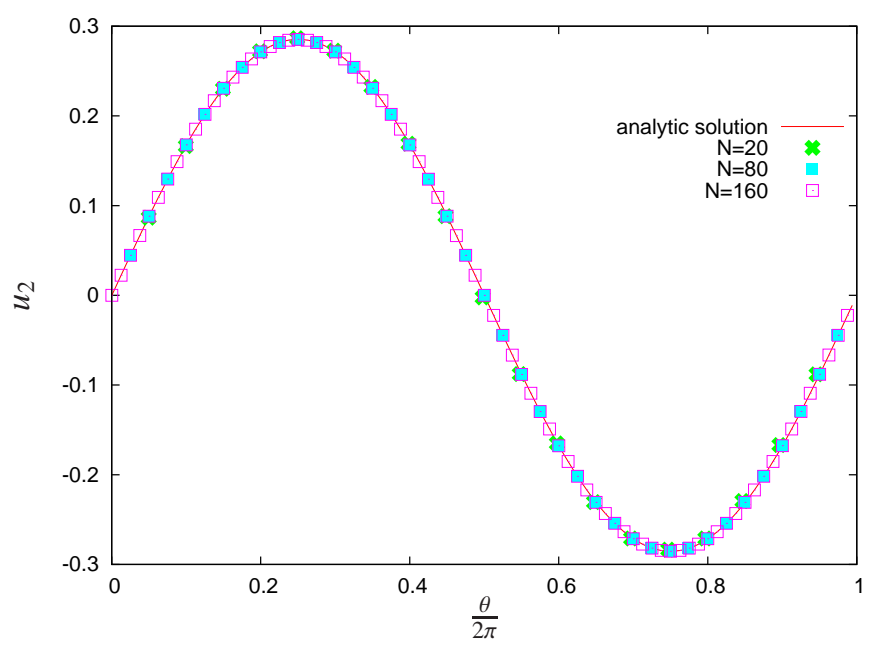

Figure 2: Reconstruction of $u_{2}$ on $\Gamma_{u}$

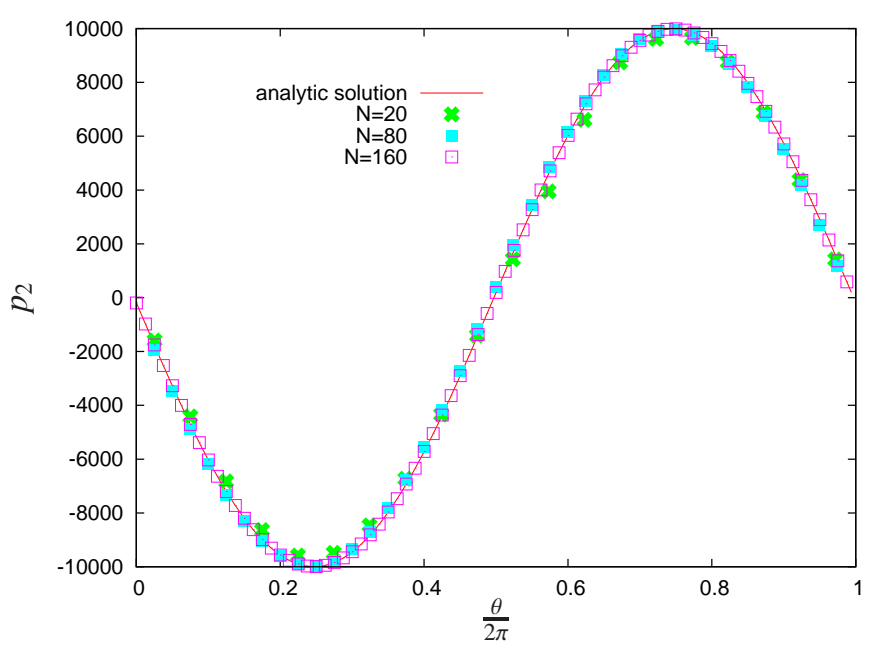

Figure 3: Reconstruction of $p_{2}$ on $\Gamma_{u}$
It is necessary to see how the reconstructions are influenced when the mesh refinement increases (depending on the number of finite elements $N$ on each boundary). Figure 2 shows the reconstructions on $\Gamma_{u}$ of the $u_{2}$ component of the displacement obtained with $N=20, N=80$ and $N=160$. Figure 3 shows the corresponding reconstructions of the $p_{2}$ component of the traction vector. Table 2 lists the errors $u_{\text {error }}$ and $p_{\text {error }}$ for different mesh refinements. It can be observed that the error level decreases as the mesh refinement increases. This confirms that the inverse method is stable with respect to the mesh refinement. It can also be observed that reconstruction using a small number of data $(N=20)$ is also accurate.

The reconstructions of the $u_{1}$ component of the displacement and of the $p_{1}$ component are not presented because the results are similar to those obtained for the $u_{2}$ and $p_{2}$ components.

\begin{tabular}{|l|l|l|}
\hline$N$ & $u_{\text {error }}$ in \% & $p_{\text {error }}$ in $\%$ \\
\hline 20 & $1.05 \mathrm{E}-3$ & $1.72 \mathrm{E}-2$ \\
\hline 40 & $6.38 \mathrm{E}-6$ & $4.00 \mathrm{E}-5$ \\
\hline 80 & $2.22 \mathrm{E}-6$ & $1.95 \mathrm{E}-5$ \\
\hline 160 & $3.20 \mathrm{E}-7$ & $4.96 \mathrm{E}-6$ \\
\hline
\end{tabular}

Table 2: influence of mesh refinement on the $u_{\text {error }}$ and on the $p_{\text {error }}$

\subsubsection{Reconstruction with noisy displacement data $\phi^{d}$}

It is necessary to see how the reconstructions are influenced when data are noisy. The noisy displacement data $\phi^{d}$ are generated by:

$$
\phi^{d}=\phi_{a n}^{d}+\delta \eta \phi_{\max }^{d}
$$

where $-1 \leq \eta \leq 1$ is a random value, $\delta$ is the noise level in $\%$ and $\phi_{\max }^{d}$ is the maximal value of the data of $\Gamma_{d}$.

The first step is to verify that the stopping criterion is still reliable when the data are noisy. The boundary parts $\Gamma_{d}$ and $\Gamma_{u}$ are both approximated with 160 finite elements. The value of parameter $c$ is arbitrarily fixed at 1 .

\begin{tabular}{|l|l|l|}
\hline noise level in \% & $u_{\text {error }}$ in \% & $p_{\text {error }}$ in \% \\
\hline 0 & $3.20 \mathrm{E}-7$ & $4.96 \mathrm{E}-6$ \\
\hline 1 & $1.96 \mathrm{E}-3$ & $8.25 \mathrm{E}-3$ \\
\hline 2 & $6.85 \mathrm{E}-3$ & $2.84 \mathrm{E}-2$ \\
\hline 3 & $1.49 \mathrm{E}-2$ & $6.11 \mathrm{E}-2$ \\
\hline 5 & $4.03 \mathrm{E}-2$ & $1.65 \mathrm{E}-1$ \\
\hline 10 & $1.50 \mathrm{E}-1$ & $5.82 \mathrm{E}-1$ \\
\hline 20 & $5.05 \mathrm{E}-1$ & 1.79 \\
\hline
\end{tabular}

Table 3: influence of the noise level on the $u_{\text {error }}$ and on the $p_{\text {error }}$

Figure 4 represents the evolution of the different control quantities $J\left(\mathbf{U}^{\mathbf{k}+\mathbf{1}}\right), J_{\Gamma_{d}}\left(\mathbf{U}^{\mathbf{k}+\mathbf{1}}\right), J_{\Gamma}\left(\mathbf{U}^{\mathbf{k}+\mathbf{1}}\right), u_{\text {error }}$ and $p_{\text {error }}$ versus the number of iterations $k$ when the noise level is at $5 \%$. It can be noticed that from the $200^{\text {th }}$ iteration the quantities $J$ and $J_{\Gamma_{d}}$ remain constant. The iterative process is stopped at this step. However if the iterations continue, it can be seen that the quantities $u_{\text {error }}$ and $p_{\text {error }}$ continue to decrease, reaching a minimum 


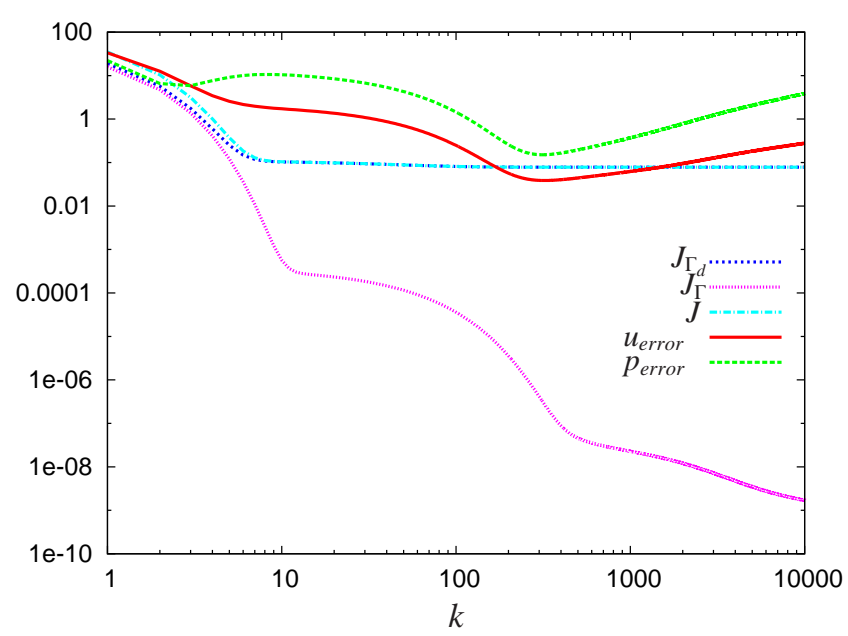

Figure 4: Evolution of control quantities versus the number of iterations $k$

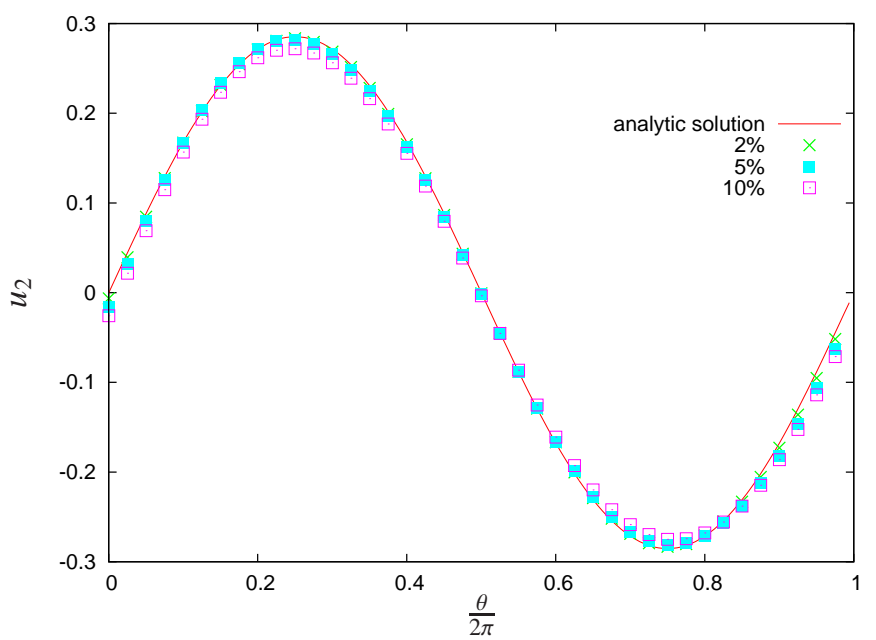

Figure 5: Reconstruction of $u_{2}$ on $\Gamma_{u}$

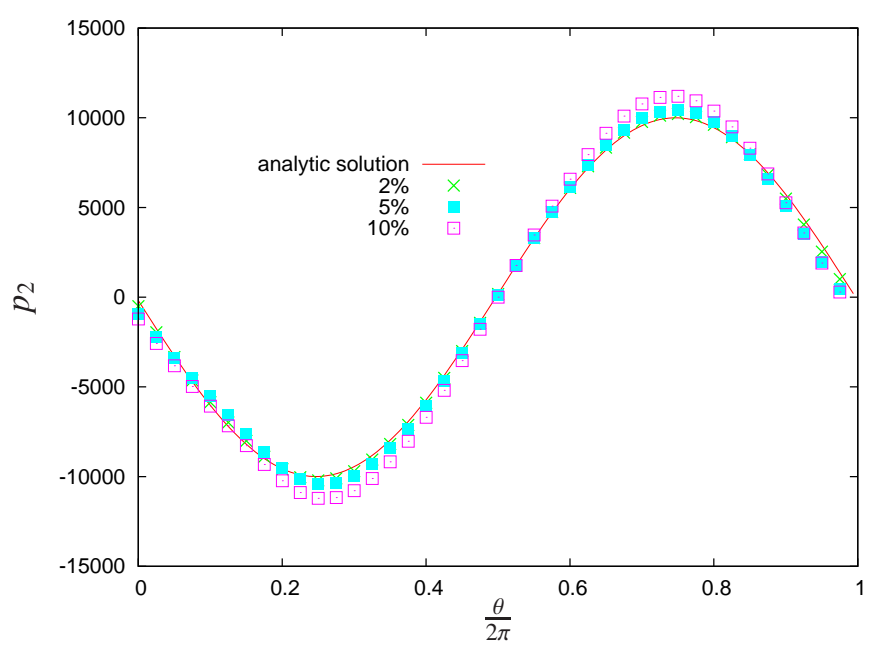

Figure 6: Reconstruction of $p_{2}$ on $\Gamma_{u}$ at around the $400^{\text {th }}$ iteration and then rising again. The stopping criterion, defined in section 5.1.1 is still valid because it leads us to choose a stable solution but it does not allow us to achieve the optimal solution (the closest to the analytical solution). The non monotonicity of the quantities $u_{\text {error }}$ and $p_{\text {error }}$ do not come out from the non compatibility part of the noise (this part of the noise is deleted because each optimal element must be compatible by definition in (8) for the continuous case and in (15) for the discrete case) but is due to the fact that the compatible data are dense in the space of all possible data. This compatible part of the noise probably leads to a different solution of the $\Gamma_{u}$ part. It is the reason why we must stop the iterative process with the L-curve criterion.

Figure 5 shows reconstructions on $\Gamma_{u}$ of the $u_{2}$ component of the displacement obtained with different noise levels $(\delta=2 \%$, $\delta=5 \%$ and $\delta=10 \%$ ). Figure 6 gives the corresponding reconstructions of the component $p_{2}$. The reconstructions obtained can be seen to be very accurate. Table 3 lists the errors on $u$ and on $p$ for the different noise levels considered.

The functional is composed of two terms with different roles. As in most inverse methods, there is a regularization term which tends to zero as the iterations go on. But, in the present method, there is also a relaxation term that allows data blurred by noise to be taken into account. We therefore seek a solution which is close to the data but not a solution that exactly fits the data. The algorithm then recomputes, at each step, a solution on the whole boundary. Figure 7 represents the reconstruction of $u_{1}$ on $\Gamma_{d}$ and the noisy data $\phi_{d}$ used $(\delta=10 \%)$. It can be noticed that the reconstruction corresponds to the analytical solution $u_{1}^{a n}$ and that the noise in the data has been deleted by the algorithm. The regularization term $J_{\Gamma}$ is negligible. The residual term $J$ is equal to the relaxation term $J_{\Gamma_{d}}$. This term corresponds to the distance on $\Gamma_{d}$ between the deblurred reconstruction and the noisy data. It can also be noted that the relations (17-19) are also verified when the data $\phi_{d}$ is noisy. It is not surprising because the inequalities were established without assumption on the data (for instance that $\boldsymbol{\Phi}^{\mathbf{d}}$ is compatible).

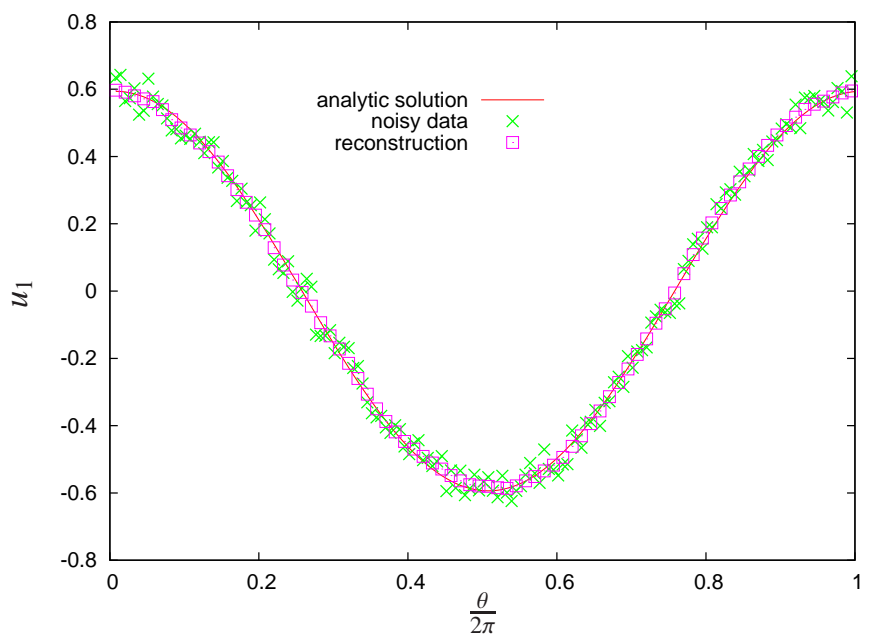

Figure 7: Noisy data $\phi^{d}$ and reconstruction of $u_{1}$ on $\Gamma_{d}$ 


\subsubsection{Reconstruction with noisy data $\psi^{d}$}

A similar study has been performed with noisy traction data. The noisy traction data $\psi^{d}$ are generated by:

$$
\psi^{d}=\psi_{a n}^{d}+\delta \eta \psi_{\max }^{d}
$$

Figure 8 represents the evolution of the different control quantities $J\left(\mathbf{U}^{\mathbf{k}+\mathbf{1}}\right), J_{\Gamma_{d}}\left(\mathbf{U}^{\mathbf{k}+\mathbf{1}}\right), J_{\Gamma}\left(\mathbf{U}^{\mathbf{k}+\mathbf{1}}\right), u_{\text {error }}$ and $p_{\text {error }}$ versus the number of iterations $k$ when the noise level is $5 \%$.

It can be noticed that from the $400^{\text {th }}$ iteration the quantities $J$ and $J_{\Gamma_{d}}$ remain constant. The iterative process will be stopped at this step. The relations (17-19) are once more verified although the data $\psi_{d}$ is noisy. It is also noticed that the quantities $u_{\text {error }}$ and $p_{\text {error }}$ reach their minimum at almost the $400^{\text {th }}$ iteration then rise again. The stopping criterion chosen in section 5.1.1 is still valid. Figure 9 gives the reconstructions of the

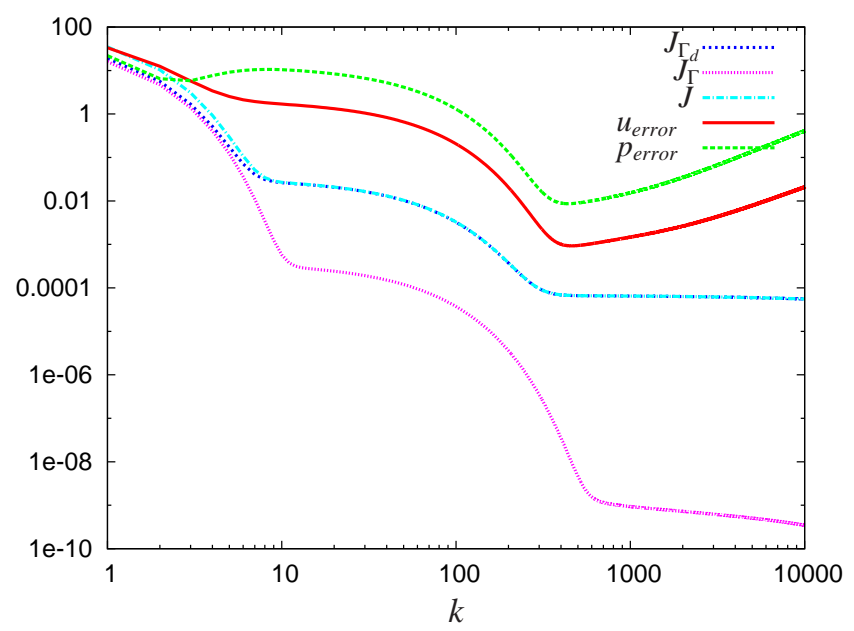

Figure 8: Evolution of control quantities versus the number of iterations $k$

component $u_{1}$ of the displacement respectively obtained with a noise level of $2 \%, 5 \%$ and $10 \%$. Figure 10 gives the corresponding reconstructions of $p_{2}$. The reconstructions obtained can be seen to be very accurate.

\subsection{Square plate under traction}

A two-dimensional isotropic linear elastic medium in a plane stress state characterized by the material constants $E=97.9$ $G P a$ and $v=0.34$ corresponding to a copper alloy has been studied. In this situation, the domain $\Omega$ is defined by:

$$
\Omega=\left\{x=\left(x_{1}, x_{2}\right) /-1<x_{1}, x_{2}<1\right\}
$$

The boundary part $\Gamma_{d}=\Gamma_{1} \cup \Gamma_{3}$ is defined by:

$$
\begin{aligned}
& \Gamma_{1}=\left\{x=\left(x_{1}, x_{2}\right) /-1<x_{1}<1, x_{2}=-1\right\} \text { and } \\
& \Gamma_{3}=\left\{x=\left(x_{1}, x_{2}\right) /-1<x_{1}<1, x_{2}=1\right\}
\end{aligned}
$$

The boundary part $\Gamma_{u}=\Gamma_{2} \cup \Gamma_{4}$ defined by:

$$
\Gamma_{2}=\left\{x=\left(x_{1}, x_{2}\right) / x_{1}=1,-1<x_{2}<1\right\} \text { and }
$$

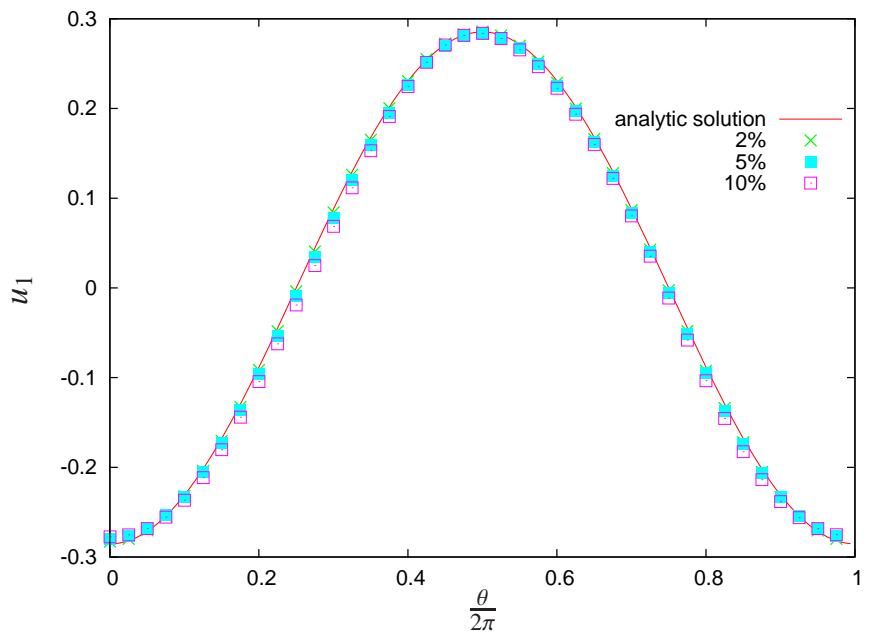

Figure 9: Reconstruction of $u_{1}$ on $\Gamma_{u}$

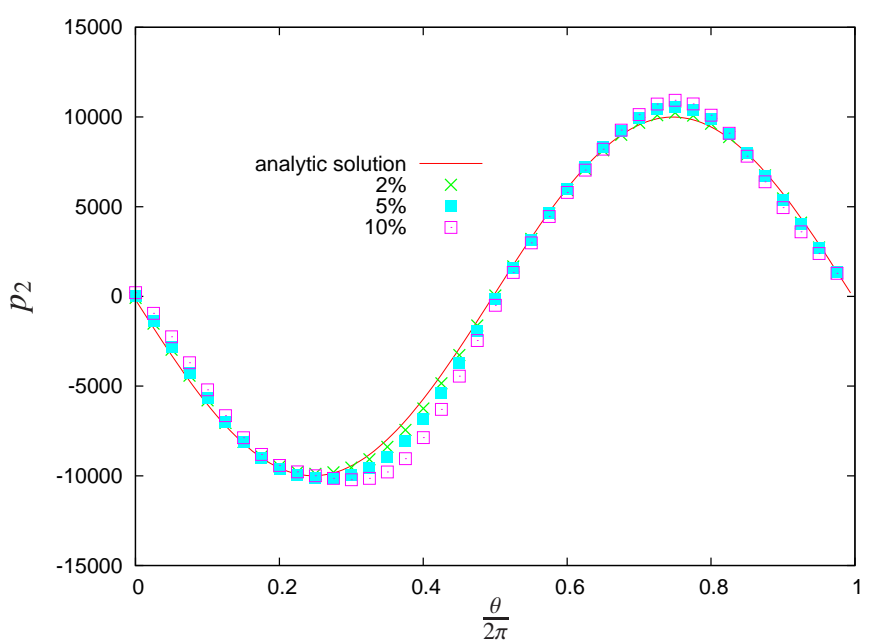

Figure 10: Reconstruction of $p_{2}$ on $\Gamma_{u}$ 


$$
\Gamma_{4}=\left\{x=\left(x_{1}, x_{2}\right) / x_{1}=-1,-1<x_{2}<1\right\}
$$

$\Gamma_{1}, \Gamma_{2}, \Gamma_{3}$ and $\Gamma_{4}$ are discretized using 100 finite elements SEG2. All finite elements have the same length and the nodes are uniformly distributed. The analytical solution under consideration for the displacements reads:

$$
u_{1}^{a n}\left(x_{1}, x_{2}\right)=\frac{1}{E} \sigma_{0} x_{1}, \quad u_{2}^{a n}\left(x_{1}, x_{2}\right)=-\frac{v}{E} \sigma_{0} x_{2}
$$

It corresponds to the uniform traction stress given by:

$$
\sigma_{11}\left(x_{1}, x_{2}\right)=\sigma_{0}, \quad \sigma_{22}\left(x_{1}, x_{2}\right)=\sigma_{12}\left(x_{1}, x_{2}\right)=0
$$

with $\sigma_{0}=15 \mathrm{GPa}$. Figure 11 gives the reconstructions of $u_{1}$ on the whole boundary $\Gamma$ when the displacement data $\phi^{d}$ is exact or blurred by a noise level of $1 \%$ and $5 \%$. Figure 12 gives the corresponding reconstructions of $p_{1}$. The reconstructions obtained are very accurate and prove the stability of the inverse method with respect to noisy data. On the boundary part $\Gamma_{d}$ supporting the data, it can be noted that the computed reconstruction is no longer noisy. Among others, the reconstructions of $p_{1}$ allow the tensile stress applied on $\Gamma_{u}$ to be identified.

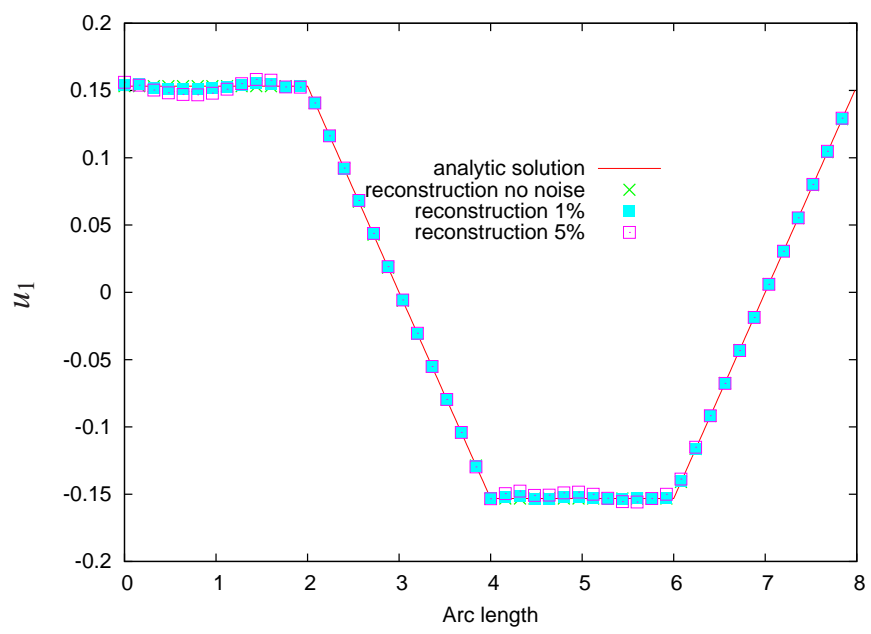

Figure 11: Reconstruction of $u_{1}$ on the whole boundary $\Gamma$

\subsection{Application of the inverse method to a contact problem}

As a final example, a contact problem without friction has been considered. An elastic cylinder with an infinite length is compressed between two parallel rigid plane supports (Figure 13). The fact the cylinder has an infinite length justifies a twodimensional study with a plane strain assumption.

The response of the elastic medium corresponding to a resin epoxy (Young's modulus $E=4 G P a$ and $v=0.4$ ) is studied. The compression load is generated by an imposed vertical displacement $u_{0}=0.002 \mathrm{~m}$ of the above support, the lower support standing still. The cylinder has a radius $R=0.1 \mathrm{~m}$.

A numerical reference solution is determined using a standard finite element method. The FEM computing code used is Cast 3m [7]. The boundary mesh used has 360 non-uniformly

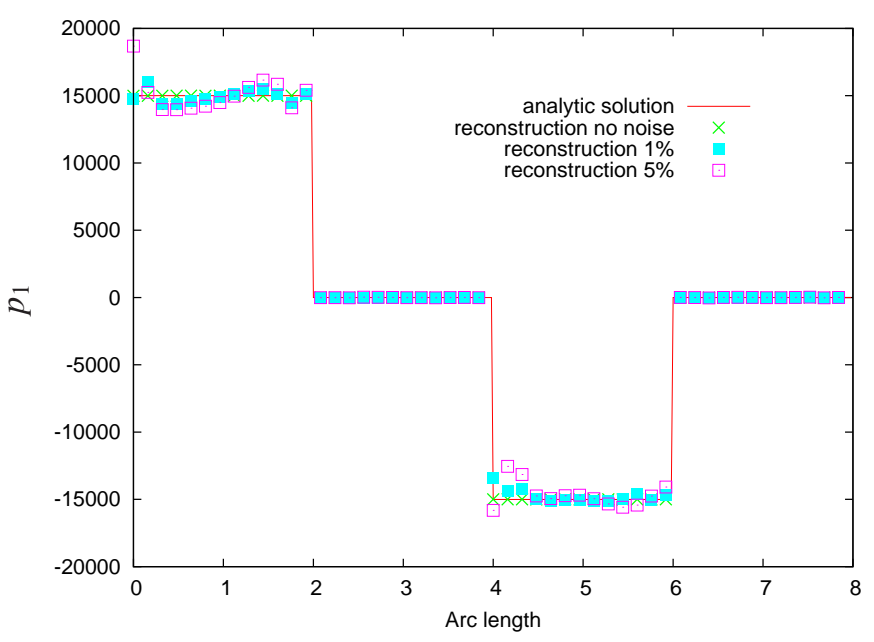

Figure 12: Reconstruction of $p_{1}$ on the whole boundary $\Gamma$

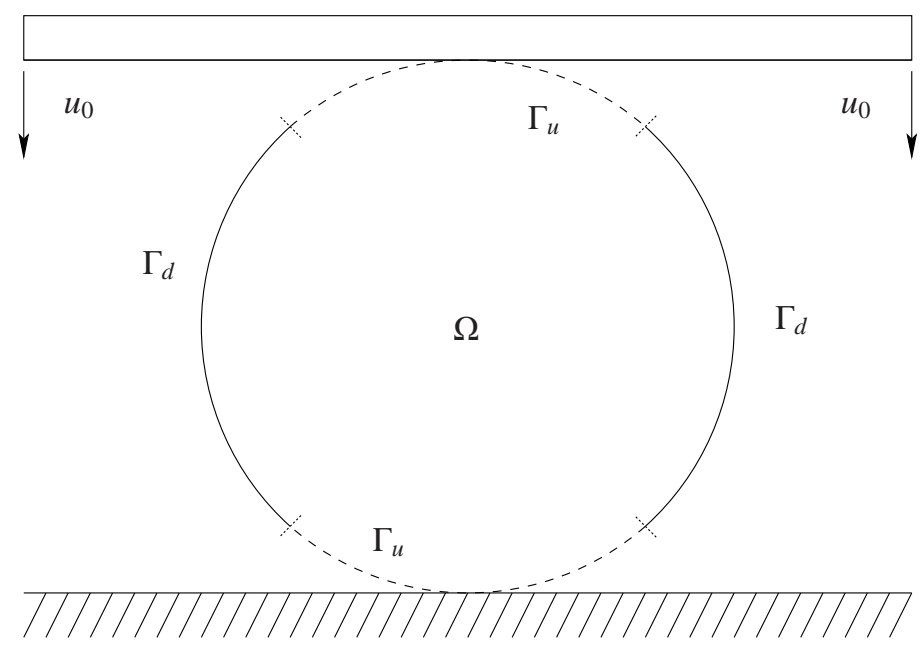

Figure 13: Definition of the domain $\Omega$

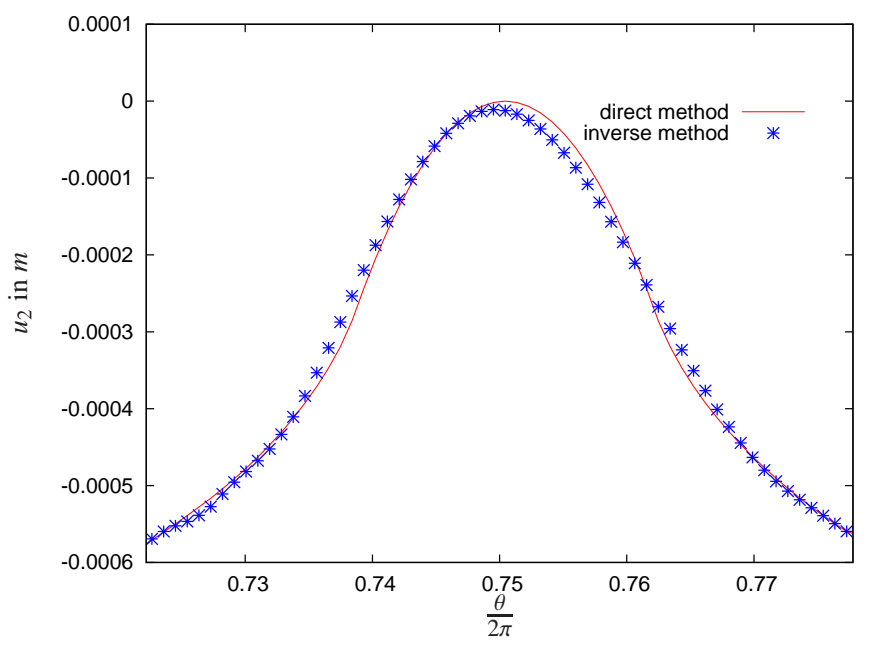

Figure 14: Reconstruction of $u_{2}$ on half of the boundary part $\Gamma_{u}$ 


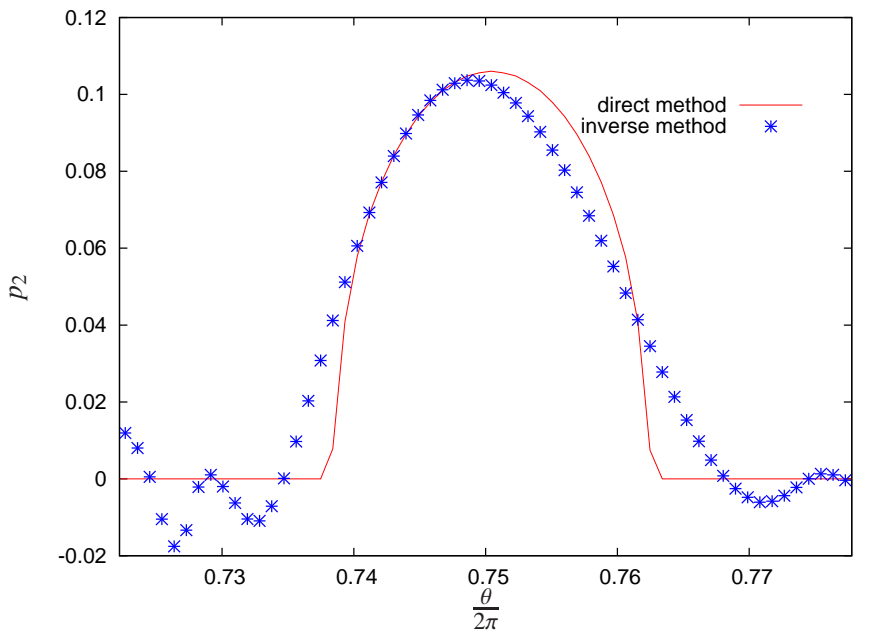

Figure 15: Reconstruction of $p_{2}$ on half of the boundary part $\Gamma_{u}$

distributed nodes, the density being higher on the $\Gamma_{u}$ part (120 uniformly distributed nodes) corresponding to $\frac{1}{8}$ of $\Gamma$.

The boundary part $\Gamma_{d}$ corresponds to $\frac{8}{9}$ of $\Gamma$ and is distributed on both sides of both contact areas (Figure 13). Noticing that $\Gamma_{d}$ is a free edge of the cylinder, given $\psi^{d}$ is zero. Data $\phi^{d}$ is the restriction to $\Gamma_{d}$ of the numerical solution obtained by the standard finite element method described above. Figures 14 and 15 compare reconstructions of the vertical displacement $u_{2}$ and the vertical nodal forces $p_{2}$ on half of the boundary $\Gamma$ with the numerical solution obtained by the finite element method. The reconstruction of the displacement (Figure 14) obtained is quite accurate and allows to identify a posteriori the extensions of the contact zones. The reconstruction of the nodal force (Figure 15) is less accurate and gives with a relative precision the maximal value of the contact pressure distribution. At the end of the boundary part $\Gamma_{u}$ some instabilities appear in the reconstruction of the vertical nodal forces.

\section{Conclusion}

This paper introduces an iterative method for solving the Cauchy problem in linear elasticity. This problem consists in recovering missing informations (displacements and forces) on some part of a boundary domain from the knowledge of overspecified data (displacements and forces) on the remaining part. This approach reduced the resolution of the Cauchy problem to the resolution of a sequence of optimization problems under equality constraints. The algorithm reads as a least square fitting. The functional is composed of two terms. The first is a relaxation term which represents the gap between the optimal element and the overspecified boundary data. The second is a regularization term which represents the gap between the optimal element and the previous optimal element. At each step, the optimal element obtained is an exact solution of the equilibrium equations and is near to the overspecified data. The regularization term vanishes when iterations go on.

Numerical simulations using the finite element method have highlighted the efficiency, accuracy and robustness of the inverse method to noisy data as well as its ability to deblur noisy data.

For all analysed situations, it can be observed that the errors in the numerical tractions obtained using the iterative method are larger than those corresponding to the reconstructed displacements. For the Cauchy problem associated with the Laplace equation, a first order method was introduced $[11,14]$ in order to improve the reconstruction of the normal derivatives. It also gave accurate reconstructions when the boundary part $\Gamma_{u}$ had corners. An extension to the Cauchy problem in linear elasticity could be useful to improve the efficiency of the inverse method presented in this paper when boundary singularities occur.

\section{7. acknowledgements}

The authors would like to thank the reviewers for their valuable comments.

\section{References}

[1] S. Andrieux, T.N. Baranger, A. Ben Abda, Solving Cauchy problems by minimizing an energy-like functional, Inverse Problems, 22, (2006), 115133.

[2] S. Andrieux, T.N. Baranger, An energy error-based method for the resolution of the Cauchy problem in 3D linear elasticity, Computer Methods in Applied Mechanics and Engineering, 197,(2008), 902-920.

[3] T.N. Baranger, S. Andrieux , An optimization approach for the Cauchy problem in linear elasticity, Structural and Multidisplinary Optimization, 35,(2008),141-152.

[4] M. Bonnet, A. Constantinescu, Inverse problems in elasticity, Inverse Problems, 21,(2005), R1-R50.

[5] L. Bourgeois, A mixed formulation of quasi-reversibility to solve the Cauchy problem for the Laplace equation, Inverse Problems, 21,(2005), 1087-1104.

[6] H.D. Bui, Fracture mechanics. Inverse problems and Solutions, Springer, Dordrecht, 2006.

[7] CASTEM 2000 (1998) Code de calcul pour l'analyse de structures par la méthode des éléments finis. Guide d'utilisation. Commissariat l'Energie Atomique, DEN/DM2S/SEMT/LM2S, F-91191 Gif-sur-Yvette, France.

[8] A. Cimetiere, F. Delvare, F. Pons, Une methode inverse avec regularisation evanescente, C.R. Acad. Sci. Paris Tome IIb, 328,(2000), 639-644.

[9] A. Cimetiere, F. Delvare, M. Jaoua, F. Pons, Solution of the Cauchy problem using iterated Tikhonov regularisation, Inverse Problems, 17, (2001), 553-570.

[10] A. Cimetiere, F. Delvare, M. Jaoua, F. Pons, An inversion method for harmonic functions reconstruction, International Journal of Thermal Sciences,41, (2002),509-516.

[11] A. Cimetiere, F. Delvare, F. Pons, Une methode inverse d'ordre un pour les problèmes de complétion de données. Comptes Rendus Mecanique, 333 (2005), 123-126.

[12] F. Delvare, A. Cimetiere, F. Pons, An iterative boundary element method for Cauchy inverse problems. Computational Mechanics, 28, (2002), 291302.

[13] F. Delvare, J.L. Hanus, Completion de donnees par methode inverse en elasticite lineaire lineaire, 7eme Colloque National en Calcul de Structures, 17-20 mai 2005, Giens, France, 2005.

[14] F. Delvare, A. Cimetiere, A first order method for the Cauchy problem for the Laplace equation using BEM, Computational Mechanics ,41,(2008), 789-796.

[15] H.W. Engl, M. Hanke, A. Neubauer, Regularization of Inverse Problems. Kluwer Academic Publications, Boston. 1996.

[16] P. Feissel, O. Allix, Modified constitutive relation error identification strategy for transient dynamics with corrupted data: The elastic case, Computer Methods in Applied Mechanics and Engineering,196, (2007), 1968-1983. 
[17] J. Hadamard, Lectures on Cauchy's problem in linear partial differential equations. Yale University Press, New Haven, 1923.

[18] P.C. Hansen, Analysis of discrete ill-posed problems by means of the Lcurve,SIAM Review,34,(1992),561-580.

[19] K.L. Johnson, Contact mechanics. Cambridge University Press, Cambridge, 1985 .

[20] M. Jourhmane, D. Lesnic, N. S. Mera. Relaxation procedures for an iterative algorithm for solving the cauchy problem for the laplace equation. Engineering analysis with boundary elements, 28, (2004), 655-665.

[21] M. V. Klibanov and F. Santosa. A computational quasi-reversibility method for Cauchy problems for Laplace's equation. SIAM Journal of Applied Mathematics, 51,(1991), 1653-1675.

[22] T. Koya, W.C. Yeih, T. Mura, An inverse problem in elasticity with partially overspecified boundary conditions. II. Numerical details. Transactions of the ASME. Journal of Applied Mechanics 60,(1993), 601-606.

[23] V.A. Kozlov, V.G. Maz'ya, A.V. Fomin, An iterative method for solving the Cauchy problem for elliptic equations, Comput. Methods Math. Phys., 31, (1991), 45-52.

[24] S. Kubo, Inverse problems related to the mechanics and fracture of solids and structures. JSME International Journal Series I, 31,(1988), 157-166.

[25] R. Lattes and J. L. Lions. Methode de quasi-reversibilite et applications. Dunod, Paris, 1967.

[26] D. Lesnic, L. Elliot, D. B. Ingham. An iterative boundary element method for solving the Cauchy problem for the Laplace equations. Engineering analysis with boundary elements, 20, (1997), 123-133.

[27] A. Maniatty, N. Zabaras,K. Stelson, Finite element analysis of some elasticity problems. Journal of Engineering Mechanics Division ASCE, 115, (1989), 1302- 1316

[28] L. Marin, L. Elliott, D.B. Ingham, D. Lesnic, Boundary element method for the Cauchy problem in linear elasticity, Engineering Analysis with Boundary Elements 25, (2001), 783-793.

[29] L. Marin, D. Lesnic, Regularized boundary element solution for an inverse boundary value problem in linear elasticity, Communications in $\mathrm{Nu}$ merical Methods in Engineering 18, (2002), 817-825.

[30] L. Marin, D. Lesnic, Boundary element solution for the Cauchy problem in linear elasticity using singular value decomposition, Computer Methods in Applied Mechanics and Engineering 191, (2002), 3257-3270.

[31] L. Marin, L. Elliott, D.B. Ingham, D. Lesnic, Boundary element regularization methods for solving the Cauchy problem in linear elasticity, Inverse Problems in Engineering, 10, (2002), 335-357.

[32] L. Marin, D.N. Hao , D. Lesnic, Conjugate gradient-boundary element method for the Cauchy problem in elasticity, Quarterly Journal of Mechanics and Applied Mathematics 55, (2002), 227-247.

[33] L. Marin, D. Lesnic, BEM first-order regularization method in linear elasticity for boundary identification, Computer Methods in Applied Mechanics and Engineering, 192, (2003), 2059-2071.

[34] L. Marin, D. Lesnic. The method of fundamental solutions for the Cauchy problem in two-dimensional linear elasticity, International Journal of Solids and Structures, 41, (2004), 3425-3438.

[35] L. Marin, A meshless method for solving the Cauchy problem in threedimensional elastostatics, Computers and Mathematics with Applications 50, (2005), 73- 92.

[36] L. Marin, D. Lesnic. Boundary element-Landweber method for the Cauchy problem in linear elasticity, IMA Journal of Applied Mathematics 18, (2005), 817-825.

[37] L. Marin, The minimal error method for the Cauchy problem in linear elasticity. Numerical implementation for two-dimensional homogeneous isotropic linear elasticity, International Journal of Solids and Structures, 46, (2009), 957-974

[38] D. Schnur, N. Zabaras, Finite element solution of two-dimensional elastic problems using spatial smoothing. International Journal for Numerical Methods in Engineering 30, (1990), 57-75.

[39] A. N. Tikhonov and V. Y. Arsenin. Solution of ill-posed problems. John Wiley and Sons, New York, 1977

[40] W.C. Yeih, T. Koya, T. Mura, An inverse problem in elasticity with partially overspecified boundary conditions. I. Theoretical approach, Transactions of the ASME Journal of Applied Mechanics 60,(1993), 595-600.

[41] N. Zabaras, V. Morellas, D. Schnur, Spatially regularized solution of inverse elasticity problems using the BEM. Communications in Applied Numerical Methods, 5, (1989), 547-553. 